\title{
Optical Absorption Enhancement in Lossy Transition Metal Elements of Plasmonic Nanosandwiches
}

Tomasz J. Antosiewicz, ${ }^{*}$ S. Peter Apell, Carl Wadell, and Christoph Langhammer* Chalmers University of Technology, Department of Applied Physics, SE-412 96 Göteborg, Sweden E-mail: tomasza@chalmers.se; clangham@chalmers.se

KEYWORDS: localized surface plasmon resonance, plasmon enhanced chemistry, palladium, optical antenna

${ }^{*}$ To whom correspondence should be addressed 


\begin{abstract}
Combination of catalytically active transition metals and surface plasmons offers a promising way to drive chemical reactions by converting incident visible light into energetic electronhole pairs acting as a mediator. In such a reaction enhancement scheme, the conversion efficiency is dependent on light absorption in the metal. Hence, increasing absorption in the plasmonic structure is expected to increase generation of electron-hole pairs and, consequently, the reaction rate. Furthermore, the abundance of energetic electrons might facilitate new reaction pathways. In this work we discuss optical properties of homo- and hetero-metallic plasmonic nanosandwiches consisting of two parallel disks made of gold and palladium. We show how near-field coupling between the sandwich elements can be used to enhance absorption in one of them. The limits on this enhancement are investigated using finite-difference time-domain simulations. Physical insight is gained through a simple coupled dipole analysis of the nanostructure. For small palladium disks (compared to the gold disk) total absorption enhancement integrated over the near visible solar AM1.5 spectrum is eight-fold, while for large palladium disks, similar in size to the gold one, it exceeds three.
\end{abstract}

\title{
Introduction
}

Discrete metallic objects, such as spheres, cubes, and disks, are capable of supporting plasmonic resonances determined by their shape, constituent materials and environments. These single particle modes are modified by the presence of other nearby structures. For example, when small dielectric objects of larger refractive index than that of the surrounding medium are placed in the vicinity of the plasmonic particle the resonance redshifts - an effect that forms the basis of surface plasmon-based sensing. ${ }^{1-4}$ If the second structure is also metallic and capable of supporting plasmons, the plasmonic entities interact and form a new set of coupled resonances. These new modes are combinations of individual resonances of the constitutive elements and, in general, are categorized as either bonding or antibonding. The simplest example is the existence of symmetric and antisymmetric surface plasmon-polaritons when two metal-dielectric interfaces are brought 
into close proximity. ${ }^{5,6}$ Mode coupling is also seen for more complex nanostructures, e.g. multiple concentric shells ${ }^{7}$ or parallel nanowires, ${ }^{8,9}$ and the frequencies of these constituents are related via sum rules. ${ }^{10}$ Metallic particles and localized surface plasmon resonances (LSPRs) have also the ability to shape the electromagnetic near-field and radiate efficiently in the far-field upon interaction with light, which makes them a key element in many applications. These include efficient light emission, ${ }^{11,12}$ photodetection, ${ }^{13,14}$ sensing, ${ }^{4,15-18}$ and enhancing chemical reactions. ${ }^{19,20}$

In general, the large local field enhancements that can be obtained can be employed for efficient polarization of an adjacent object like a molecule or a second nanoparticle. If that particle is also a metal or a semiconductor, the induced stong plarization may boost energetic electron (e-h pair) creation in the particle and, for example, subsequent injection into adsorbates. A second potentially interesting effect is the coupling of the energetic electrons to lattice phonons and the subsequent use of the particle as a localized heat source for, e.g. a temperature-controlled catalytic reaction. The last two cases are, mechanistically, connected to maximization of light absorption in an appropriate material. This, in turn, relates to a more profound and fundamental question of what is the optimum geometrical arrangement and material combination to maximize a physical parameter such as optical absorption, given a certain (constant) amount of material present. ${ }^{21}$ This is one of the main issues we address in this article since in the specific case of plasmon-enhanced chemical reactions, ${ }^{19,20,22}$ efficient light absorption is the first key step. Subsequently, the plasmon acts as a mediator either donating energetic electrons to LUMO orbitals of available adsorbates ${ }^{19,20,23-26}$ or by providing thermal energy by constituting a local heat source. A first experimental manifestation of the generic absorption engineering strategy discussed here, was recently demonstrated by Wadell et al. $^{27}$

Naturally, absorption engineering as presented here is not only limited to increasing e-h pair generation for driving a chemical reaction. A parallel research track utilizes absorption also for sensing applications. For example, periodically arranged Au nanodisks or Pd nanowires placed on top of a dielectric spacer above a thick gold film achieve $c a .99 \%$ absorption. ${ }^{28,29}$ This almost perfect absorption owes its existence to electromagnetic coupling of the top metal pattern to the 
bottom Au film. Thus, a slight change of the conditions, e.g. brought about by a change of the refractive index of the surrounding material, causes the absorption resonance to shift. As a result, the reflection signal measured at the original resonance wavelength undergoes an easily detectable increase. Therefore, these so-called "perfect absorbers" (at a single wavelength) offer a robust sensing method characterized by a large figure-of-merit.

Here we investigate the generic concept of maximization of surface plasmon mediated light absorption by studying a model system composed of two parallel metallic disks separated by a dielectric spacer layer and by varying systematically their size (see Fig. 1). Since the main objective of this work is to identify and understand absorption enhancement effects, we compare throughout this study quantitatively the power loss in a coupled bi-particle system to that in single relevant particles of the same size. In finite-difference time-domain (FDTD) simulations for these systems we find up to eight-fold increase of total (integrated over a wide wavelength range from 280 to 1200 $\mathrm{nm}$ and utilizing the solar AM1.5 spectrum ${ }^{30}$ ) absorption in a palladium (Pd) nanodisk placed on top of a gold $(\mathrm{Au})$ disk. We also conduct a qualitative analysis by means of a coupled dipole model to understand the physics of the enhancement.

\section{Plasmonic nanosandwiches}

We consider a system composed of two plasmonic nanodisks placed one on top of the other (sandwich), as shown in Fig. 1. Such structures are supported by substrates, so access of molecules to the bottom disk is restricted and chemical reactions will therefore predominantly occur on the surface of the top disk, which is easily accessible. Thus, the top disk for our study is either Au or $\mathrm{Pd}$, while the bottom one is always Au. Notice, that the studied plasmonic system is representative for any arrangement of coupled particles in which one element is composed of a noble metal with low losses and the other of a metal characterised by large dissipation, e.g. a catalytic transition metal. The radii and permittivities of the two disks are $r_{b, t}$ and $\varepsilon_{b, t}$, respectively, with $b, t$ indicating bottom and top, respectively (see Fig. 1 for details). The thickness $h=20 \mathrm{~nm}$ of both disks 
is the same and they are separated by a surface-to-surface distance $d \in\langle 0 ; 30\rangle \mathrm{nm}$, and placed in vacuum. Linearly polarized light is incident from the top with the wavevector perpendicular to the surface of the disks.

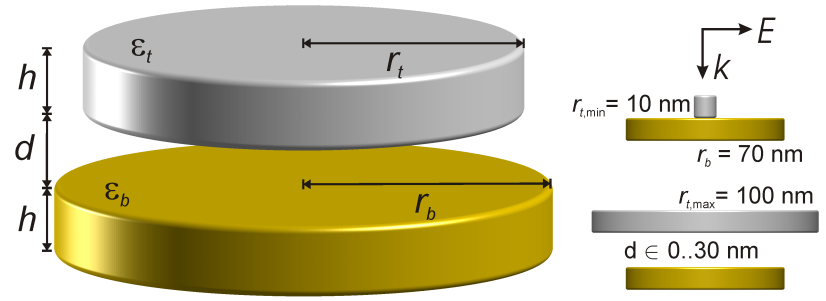

Figure 1: Plasmonic nanosandwich composed of two disks. The disks are made of material with permittivity $\varepsilon_{b, t}$ and their radii are $r_{b, t}$ with $b, t$ indicating bottom and top, respectively. The disk thickness is $h=20 \mathrm{~nm}$, surface separation is $d$, and they are placed in vacuum. On the right we show variation of the top radius $r_{t}$ from $10-100 \mathrm{~nm}$ and disk separation $d$ from $0-30 \mathrm{~nm}$.

Simulations are performed using the FDTD method (commercial algorithm ${ }^{31}$ ) with a nonuniform mesh and the smallest spatial discretization step is $1 \mathrm{~nm}$. The presented optical extinction, scattering and absorption spectra are calculated by dividing the respective cross sections by the projected area of the nanosandwich. ${ }^{32}$ In this way, when the top disk is smaller than the bottom one $\left(r_{t} \leq r_{b}=70 \mathrm{~nm}\right)$ the cross sections are divided by $\pi r_{b}^{2}$ and when it is larger by $\pi r_{t}^{2}$, i.e. surface area of the larger of the two disks. Material data for $\mathrm{Au}$ and $\mathrm{Pd}$ is taken from the work of Johnson and Christy. ${ }^{33,34}$

For the remainder of our article, we first discuss the case of Au-Au nanosandwiches as our "benchmark" system ${ }^{35}$ to be able to present clearly changes brought on by substituting a Pd nanodisk for a Au one. We then go on to a heterometallic system consisting of a Au bottom and Pd top disk and analyze in detail the local light absorption in this system based on FDTD simulations. Finally, we provide a quantitative understanding of the physics in these systems by means of the coupled dipole approximation.

\section{Au-Au nanosandwich}

We start our investigations with a homometallic nanosandwich, for which $\varepsilon_{b}=\varepsilon_{t}$. Figure 2 shows optical efficiency spectra of a homometallic nanosandwich composed of Au with a fixed bottom 
radius $r_{b}=70 \mathrm{~nm}$ and a varied top $r_{t} \in\langle 10,100\rangle \mathrm{nm}$. Since the disk separation, $d=15 \mathrm{~nm}$, is relatively small compared to $r_{b}$, the disks are relatively strongly coupled as evident from the optical spectra. Light incident perpendicularly to the surface of a disk induces a dipolar mode which interacts with the induced dipole of the second disk. The coupling between them results in the appearance of two new modes - one where the the dipole moments are aligned parallel to each other (this is the short wavelength maximum seen in extinction for $r_{t}>40 \mathrm{~nm}$ in Fig. 2), and one antiparallel - the long wavelength one, which starts for $r_{t}=0 \mathrm{~nm}$ at $650 \mathrm{~nm}$ and then as the radius increases bends towards larger $\lambda$. This differentiation becomes apparent after comparing the extinction (a) and phase shift (b) plots, where the phase shift plot shows the phase difference of the electric field in the center of the nanodisks.

The short wavelength maximum is associated with an approximately negligible phase shift between the disks corresponding to in-phase dipoles. The second resonance band follows a $\pi$ phase shift, which means that the disks are in antiphase. The in-phase mode, starting at $r_{t} \approx 40 \mathrm{~nm}$, is dipolar as evident by dominant scattering for large (radius $70 \mathrm{~nm}$ ) structures. ${ }^{36,37}$ On the other hand, for the anti-phase mode the scattering efficiency (Fig. 2c) drops to almost zero when the top disk radius matches the bottom one $(c a .70 \mathrm{~nm})$ as the nanosandwich behaves like a quadrupole (two anti-parallel dipoles).

In the arrangement considered here, where the top disk radius is changed, a large phase shift between the disks is not only observed for the bonding hybridized mode with weak scattering, but also when $r_{t}<40 \mathrm{~nm}$. This can be rationalized easily since with a decreasing top disk radius coupling in the nanosandwich weakens and the dipolar resonance of the bottom disk dominates the spectrum at about $650 \mathrm{~nm}$. The induced field of the bottom disk now polarizes the smaller one completely giving it an effective phase shift, rather than hybridizing with it. A similar effect can be seen when top disk increases beyond the size of the bottom one. There, the coupled mode becomes increasingly dipolar, as made evident by quick increase of scattering (see Fig. 2c).

We now turn to the individual absorption efficiencies of the bottom, $Q_{\mathrm{a}}^{b}$, and top, $Q_{\mathrm{a}}^{t}$, disks shown in Fig. 2e and $\mathrm{f}$, respectively. For $r_{t}<30 \mathrm{~nm}$ practically all dissipation occurs in the 

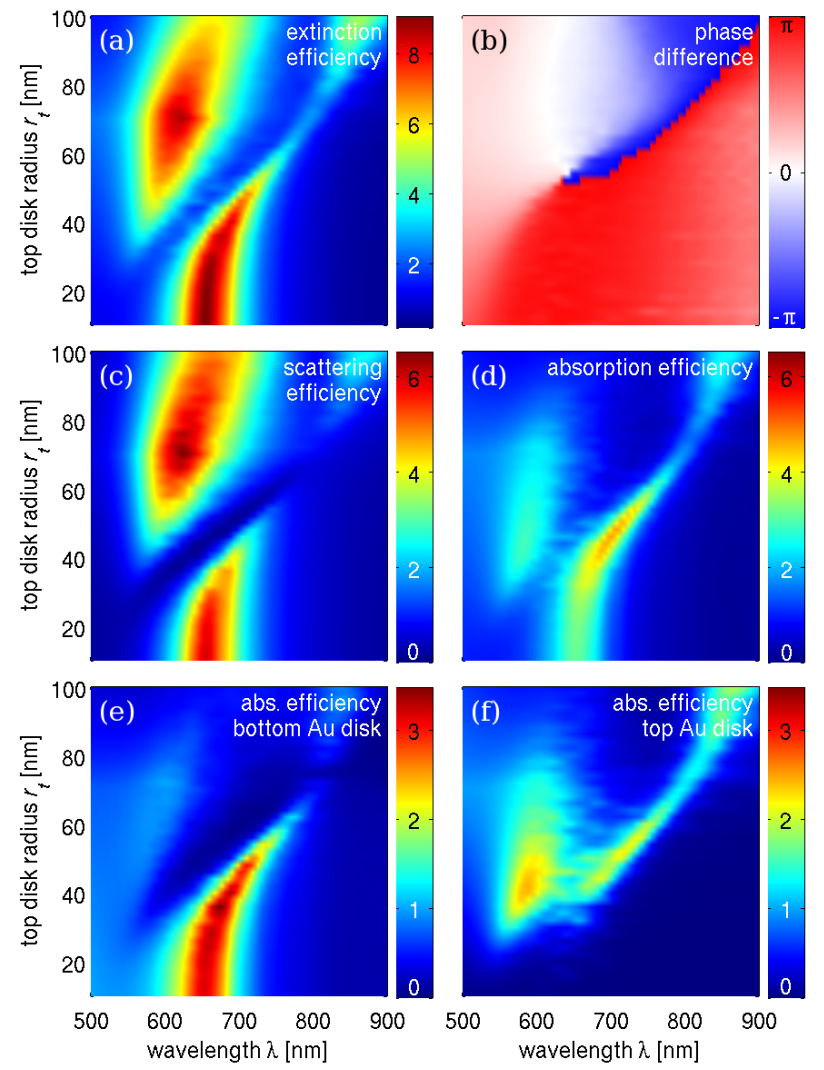

Figure 2: Optical properties of a gold-gold nanosandwich for widely varying top disk radius: (a) extinction, (b) phase shift between disks as measured in their geometrical centers, (c) scattering, (d) total absorption, (e) absorption in the bottom disk, and (f) absorption in the top disk. The disk spacing is $d=15 \mathrm{~nm}$ and the bottom radius $r_{b}=70 \mathrm{~nm}$. The scale is the same in (c), (d) and in (e), (f). Coupling of the two Au disks gives rise to a short wavelength antibonding and a long wavelength bonding mode. The former is connected to in-phase oscillations in the disks, while the latter corresponds to oscillations in anti-phase. For the antibonding mode, absorption is dominant in the top disk - note the resonance with a maximum at ca. $600 \mathrm{~nm}$ and for $r_{t}=40 \mathrm{~nm}$ in (f) while for the bonding mode the absorption balance shifts from the bottom (dominates for $r_{t} \lesssim 60$ $\mathrm{nm}$, see the bent resonance band in (e) up to $c a .750 \mathrm{~nm}$ ) to the top disk (see (f) for the narrow resonance band starting at $700 \mathrm{~nm}$ ) for increasing $r_{t}$. 
bottom disk, since the sandwich is weakly coupled and absorption is proportional to the particle volume. Interesting effects begin when $r_{t}$ becomes large enough for the disks to couple efficiently and absorption starts to increase in the top disk. At the short wavelength resonance the top disk dominates the total absorption spectrum by accounting for about $\frac{2}{3}$ of absorption of the whole structure. In other words, absorption in the top disk is much larger than determined by its volume alone. Hence, the balance of absorption is shifted towards the top disk.

The top-to-bottom disk absorption ratio at the low energy (bonding) resonance is different, but again depends on their relative size. Due to the relative phases of the incident and induced fields, the balance is shifted towards a smaller top radius, ${ }^{38}$ and absorption peaks of equal amplitude are observed for $r_{t} \approx 54 \mathrm{~nm}$ (for a separation of $d=15 \mathrm{~nm}$ ). Consequently when $r_{t} \lesssim 54$ absorption dominates in the bottom disk, whereas for $r_{t} \gtrsim 54$ it dominates in the top one.

\section{Au-Pd nanosandwich}

We now change the top disk to Pd, which has a much broader and weaker resonance than $\mathrm{Au}, 37,39$ and keep all other parameters the same. The corresponding optical spectra are shown in Fig. 3. Clearly, the coupling of the $\mathrm{Au}$ and $\mathrm{Pd}$ disks is different than in the previous case. For a given top disk radius $r_{t}$ only one well-defined resonance can be seen and absorption in the top disk is altered considerably (compare Fig. 3f - Pd and Fig. 2f - Au) The absorption spectrum of the bottom Au disk (Fig. 3e) in the heterometallic sandwich is, however, very similar to the bottom disk of the $\mathrm{Au}-\mathrm{Au}$ structure (Fig. 2e). The narrow peak in extinction for $r_{t}<60 \mathrm{~nm}$ is reminiscent of the one observed for the homo-material case, which means that the Au-Pd system for small Pd disks is, as expected, dominated by the optical spectrum of the Au disk. However, weak coupling between the disks is present, as the peak undergoes a slight redshift with increasing $r_{t}$. During this redshift, it decreases in amplitude at the expense of increased absorption efficiency $Q_{\mathrm{a}}^{\mathrm{Pd}}$ in $\mathrm{Pd}$. We note here, that maximum $Q_{\mathrm{a}}^{\mathrm{Pd}}$ is comparable to maximum $Q_{\mathrm{a}}^{\mathrm{Au}}$, while in the homometallic $\mathrm{Au}-\mathrm{Au}$ sandwich absorption in the top disk is considerably lower than in the bottom one.

Comparing the total absorption efficiency of the Au-Au and Au-Pd systems we notice an im- 

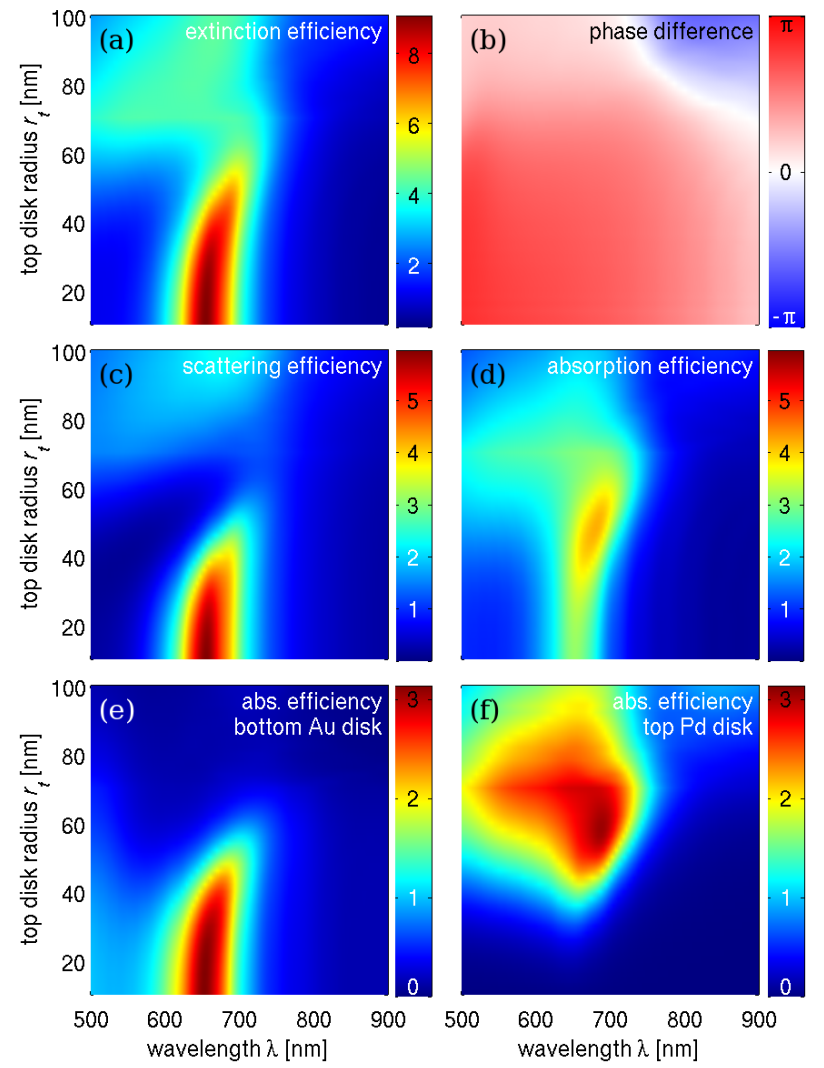

Figure 3: Optical properties of a gold-palladium nanosandwich: (a) extinction, (b) phase shift between disks as measured in their geometrical centers, (c) scattering, (d) total absorption, (e) absorption in the bottom Au disk, and (f) absorption in the top Pd disk. The disk spacing is $d=15 \mathrm{~nm}$ and the bottom radius $r_{b}=70 \mathrm{~nm}$. Note the same color scale in (c), (d) and in (e), (f). Coupling of the $\mathrm{Au}$ and Pd disks does not show clear distinction into antibonding and bonding modes since resonances in extinction do not follow 0 - and $\pi$-phase shifts. We rather see that the optical properties of the nanosandwich are determined mainly by the Au disk for a small $r_{t}$ and by the Pd disk for a large $r_{t}$. When the disks are similar in size $\left(r_{t} \approx 60 \div 70 \mathrm{~nm}\right)$ both disks are driven by the incident field with similar efficiency. At shorter wavelength light couples predominantly to the Pd disk only (note the strong scattering and absorption by the top Pd disk) and at longer wavelength the Au disk is mostly excited. It couples, however, very efficiently to the Pd disk and drives it to, consequently, increasing the absorption efficiency in Pd to three. 
portant difference, namely a lack of distinct bonding and antibonding resonances for the latter case. The extinction maxima do not correlate with 0 - and $\pi$-phase shifts in the same way as for the Au-Au case.

When the Pd disk is small in comparison to the Au disk, the latter determines the optical properties of the nanosandwich. However, for a reversed radius ratio, the Pd disk becomes the dominant one. Interestingly, the only similarity to the homometallic case is the resonance observed for small top disk radii $r_{t}$, which is the result of a weak interaction of the bottom Au disk with the small top one. This is seen in Fig 3e, where absorption occurs predominantly in the bottom disk. When the Pd disk becomes larger, absorption is concentrated in it, however, the absorption maximum is spectrally quite broad in contrast to that in Au.

The reason behind these findings is an overlap of two modes. The first one is the result of coupling between the Au and Pd disks with the former driving the latter. It occurs at an extrapolated curve from the maximum absorption efficiency in the Au disk, as seen in Fig. 3e,f. The coupling between the disks is quite efficient since Pd absorption reaches 3 with $\mathrm{Au}$ absorption being less than 1. This resonance is relatively narrow due to originating at the Au disk. The second resonance is a mostly Pd disk resonance which, as such, is much broader than the Au-induced one. Consequently, it overlaps with the first one described above. It starts to be seen at $r_{t}=70 \mathrm{~nm}$ and shows a steady redshift with an increasing $r_{t}$. Since it is mostly concentrated in the Pd disk, we expect it to be mainly dipolar and have a considerable scattering efficiency, as confirmed in Fig. 3c.

\section{Absorption enhancement: Comparison with single disks}

As noted in the introduction, our main interest in this work is to scrutinize absorption enhancement in a plasmonic nanoparticle resulting from placing it above a second one, which acts as an optical antenna for energy transfer. On the background of the comprehensive analysis of the involved resonances presented above, we define absorption enhancement (also referred to as only enhancement) as the ratio of the absorption cross section calculated for the constituent disks of the nanosandwich 


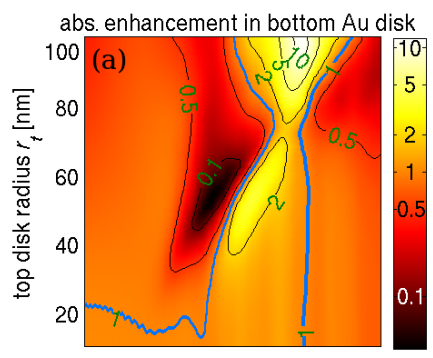

abs. enhancement in bottom Au disk

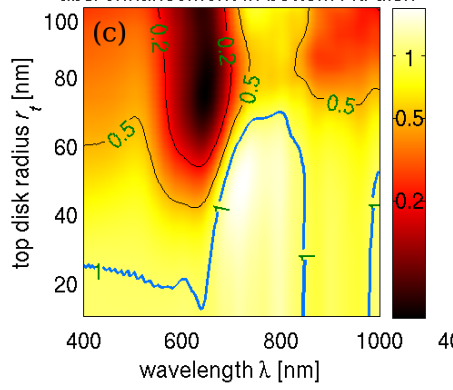

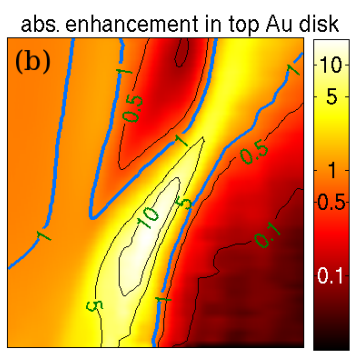

abs. enhancement in top Pd disk

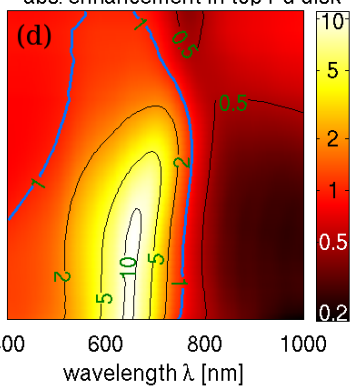

Figure 4: Absorption enhancement (absorption cross section in nanosandwich elements normalized to absorption in a lone $\mathrm{Au}$ or Pd disk) in top and bottom disks in homometallic (a,b) and heterometallic $(\mathrm{c}, \mathrm{d})$ nanosandwiches, respectively. Coupling in the nanosandwich causes a large increase of absorption in the smaller of the two disks in the Au-Au structure and it is especially high at the out-of-phase resonance. In the Au-Pd case large absorption enhancement in the top Pd disk is due to the Au nanoantenna coupling energy into the small Pd disk, which acts like a receiver. The pseudo-color scales are logarithmic, the blue line indicates enhancement of unity (equal absorption in single disk and in top disk in sandwich), and the disk separation is $d=15 \mathrm{~nm}$ and $r_{b}=70 \mathrm{~nm}$. 
and respective cross sections for single disks of the same radius. The results of such an analysis are plotted in Fig. 4 with (a,b) showing the data for homometallic and (c,d) the heterometallic structures.

Enhancement values span a slightly larger range in the $\mathrm{Au}-\mathrm{Au}$ structure than in the $\mathrm{Au}-\mathrm{Pd}$ one. This is to be expected, since the resonance bands in the first case are narrow, especially the bonding long wavelength one, and slight changes to the spectrum give thus large changes of the ratio. Further, between the bonding and antibonding modes for $\mathrm{Au}-\mathrm{Au}$ an absorption minimum is present (stemming from the bottom disk spectrum with the efficiency going almost to zero). This gives a relatively large variation of absorption enhancement in both disks.

For our continued analysis we focus now mainly on absorption in the top disk which, under experimental conditions, is fully accessible to the environment. In this way it may act, for example, as a catalytically active surface whose activity might be promoted by enhanced light absorption (eh pair generation and/or heat) and/or enhanced fields (polarization).

Large absorption enhancement in the top disk (Fig. 4b) follows predominantly the bonding resonance and reaches a maximum of $10+-$ fold at $r_{t} \approx 40 \mathrm{~nm}$. Additionaly, we observe a weaker enhancement band which is the result of the antibonding resonance in the short wavelegnth part of the spectrum. Beyond the bonding mode, in the red part of the spectrum, absorption in the top disk decreases quickly to less than 0.1 of the corresponding lone disk since the bonding resonance band is quite narrow and long wavelengths do not contribute greatly to total absorption. However, at shorter wavelengths the ratio is larger than unity for a broad spectral range $(\lambda \in\langle 400,700\rangle \mathrm{nm})$.

Absorption enhancement in the heterometallic sandwich is shown in Fig. 4c,d and is largest at the resonance of the Au disk up to a radius of about $60 \mathrm{~nm}$. It spectrally extends up to $750 \mathrm{~nm}$. In this regime the Au disk behaves like a nanoantenna which couples radiation from incident light into the top Pd disk. The striking and most interesting feature of this arraangement is that the spectral position of the maximum enhancement can be tuned by changing the size of the driving Au disk. In this way, by finding the optimum value of $r_{b}$, the absorption maximum can, for example, be matched with the spectrum of incident light, e.g. sunlight. 
We enter a new regime when the Pd disk radius becomes comparable to that of the Au disk. The absorption enhancement in the Pd disk drops below 2 because the Au antenna ceases to drive the top disk. Hence, without this coupling for $r_{t}>80 \mathrm{~nm}$ the palladium disk in the sandwich has an enhancement greater than one only in a narrow wavelength range and altogether absorbs less energy than if it were alone, as is discussed further below.

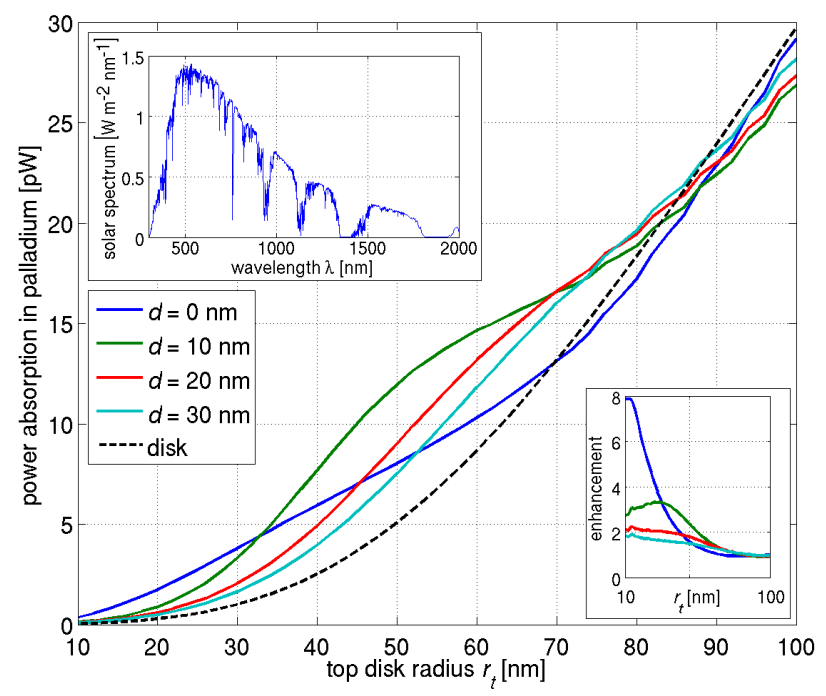

Figure 5: Total absorption in Pd vs. Pd disk radius for heterometallic nanosandwiches with $d=0$, 10, 20, ad $30 \mathrm{~nm}$ (continuous lines) and a single Pd disk as reference (dashed line). The top-left inset shows the AM1.5G solar spectrum used as the source. ${ }^{30}$ The bottom-right inset shows the total absorption enhancement. Absorption enhancement in a small Pd disk is the largest when the disks are touching and decreases as the disks are moved away from each other. For all Pd disk radii that are smaller than the Au disk the Pd disk in the sandwich absorbs more than a lone one by itself and only when its radius becomes larger than about $85 \mathrm{~nm}(d \geq 10)$ does the enhancement drop below one.

Before we start out with a more detailed analysis of the enhancement mechanisms in the different regimes, from an application point of view, it is interesting to consider the fact that absorption enhancement, as discussed above, is a parameter that is specific for a single wavelength. It is therefore also relevant to calculate absorption for a wider spectral range and to match the enhancement to the spectral intensity distribution of the used light source. Since, e.g., for potential large scale application of photocatalysis, the most abundant light source is the sun we use the AM1.5G solar spectrum ${ }^{30}$ (see inset in Fig. 5) to calculate total absorption (integrated over all wavelengths considered, that is $280-1200 \mathrm{~nm}$ ) in only the top Pd disk and compare it to that of a solitary one. 
The results, presented in Fig. 5, are calculated for a number of separation distances $d$ and clearly show that significantly more power is absorbed in the Pd disk coupled to a bottom Au antenna than when the $\mathrm{Pd}$ disk is alone. Maximum enhancement of 8 is obtained for $r_{t}=10 \mathrm{~nm}$ and the $\mathrm{Pd}$ lying directly on the gold disk. The superior performance of this arrangement is easily understandable, since it corresponds to a large antenna driving a much smaller one with an electric field of much higher amplitude than the incident field. Furthermore, such a small Pd disk has maximum absorption in the UV, where the irradiated power from the sun rapidly decreases. Hence, for small Pd disks the Au nanoantenna serves an additional role of enhancing the driving field at visible wavelengths, where the intensity is much greater than in the UV range.

When the disks are moved further away from each other the field (from the Au antenna) driving the Pd one decreases and we expect the enhancement to be smaller. Indeed, for a separation of 10 nm simulations show maximum enhancement of only $c a .3 .5$ for $r_{t}=30 \mathrm{~nm}$ which drops to a two-fold increase at $r_{t}=55 \mathrm{~nm}$. Further increase of the Pd disk causes the enhancement to drop even more and for equal disk sizes for the Au and Pd the enhancement is on the order of 1.25. Only for radii greater than $85 \mathrm{~nm}$ does the Pd single disk absorb more light than the one in the sandwich.

\section{Coupled dipole analysis of nanosandwiches}

Having shown in FDTD simulations quantitative absorption enhancement in the palladium top disk of a nanosandwich we now use the coupled dipole approximation (CDA) ${ }^{40,41}$ to analyze qualitatively the physical origin of the made observations in the simulations. We show that the found absorption enhancement is a result of transferring energy from the bottom disk to the top one. Details on the CDA may be found in Ref. ${ }^{40,41}$

Here, for a simple analysis of the nanosandwiches we model each disk using only one dipole placed at their respective geometrical centers. We assume, that in the quasistatic limit, the polariz-

ability of each dipole is $\alpha^{\mathrm{q}}=\frac{V}{4 \pi}\left(\varepsilon_{m}-\varepsilon_{s}\right) /\left(\varepsilon_{s}+L\left(\varepsilon_{m}-\varepsilon_{s}\right)\right)$, where $\varepsilon_{m}$ and $\varepsilon_{s}$ are the permittivities of the metal particle and surrounding medium, respectively, $V$ is the particle volume, and $L$ is a 
depolarization factor dependent on particle shape and orientation. ${ }^{32}$ Due to the varying size of the disks from small (radius $10 \mathrm{~nm}$, constant thickness $20 \mathrm{~nm}$ ) to quite large (radius up to $100 \mathrm{~nm}$ ) we include dynamic depolarization and radiative damping. This modifies the static polarizability $\alpha^{\mathrm{q}}$ to an effective one, which is described by the modified long wavelength approximation (MLWA) ${ }^{42,43}$ $1 / \alpha^{\mathrm{M}}=1 / \alpha^{\mathrm{q}}-\frac{2}{3} i k^{3}-\frac{k^{2}}{a}$, where $k=2 \pi / \lambda$ is the wavenumber of exciting light of wavelength $\lambda$ and $a$ is a length associated with the particle geometry.

We limit our dipole analysis to the Au-Pd nanosandwich, as this is the main structure of interest here. We note, however, that our analysis is generic and applicable to other transition metals than Pd that feature relatively high losses in the near visible spectral range. Solving the two coupled equations for the polarizabilities $\mathbf{P}_{i}$ of the two disks in Eqs. (1) and (2)

$$
\begin{aligned}
& \mathbf{P}_{1}=\alpha_{1}\left(\mathbf{E}_{0} e^{i k z_{1}}-\mathbf{A}_{12} \cdot \mathbf{P}_{2}\right), \\
& \mathbf{P}_{2}=\alpha_{2}\left(\mathbf{E}_{0} e^{i k z_{2}}-\mathbf{A}_{21} \cdot \mathbf{P}_{1}\right),
\end{aligned}
$$

where $\mathbf{A}_{i j}$ determines dipole radiation (Eq. (5)), we focus on the absorption and extinction. Interest in the former is natural in the context of our work, while the latter shows the reason behind the increased absorption efficiency in the top palladium disk.

Figure 6 presents optical efficiencies of an Au-Pd nanosandwich modeled using CDA. In the first four subfigures a-d we show the total quantities together with the phase difference between induced dipole moments to show qualitative similarity between the CDA spectra and those obtained using FDTD in Fig. 3. The main difference here is stronger coupling between the disks predicted by the dipole model than by FDTD, as seen by much stronger redshift of the single resonance for an increasing radius.

The main result is shown in Fig. 6e-h with e,f presenting absorption, and g,h extinction, in both of the disks. In both cases the first of the two plots corresponds to the bottom Au disk. The qualitative agreement for absorption obtained by CDA and FDTD is very good. The bottom Au disk absorbs most of the incident radiation when its radius is much larger than the Pd one. Then 

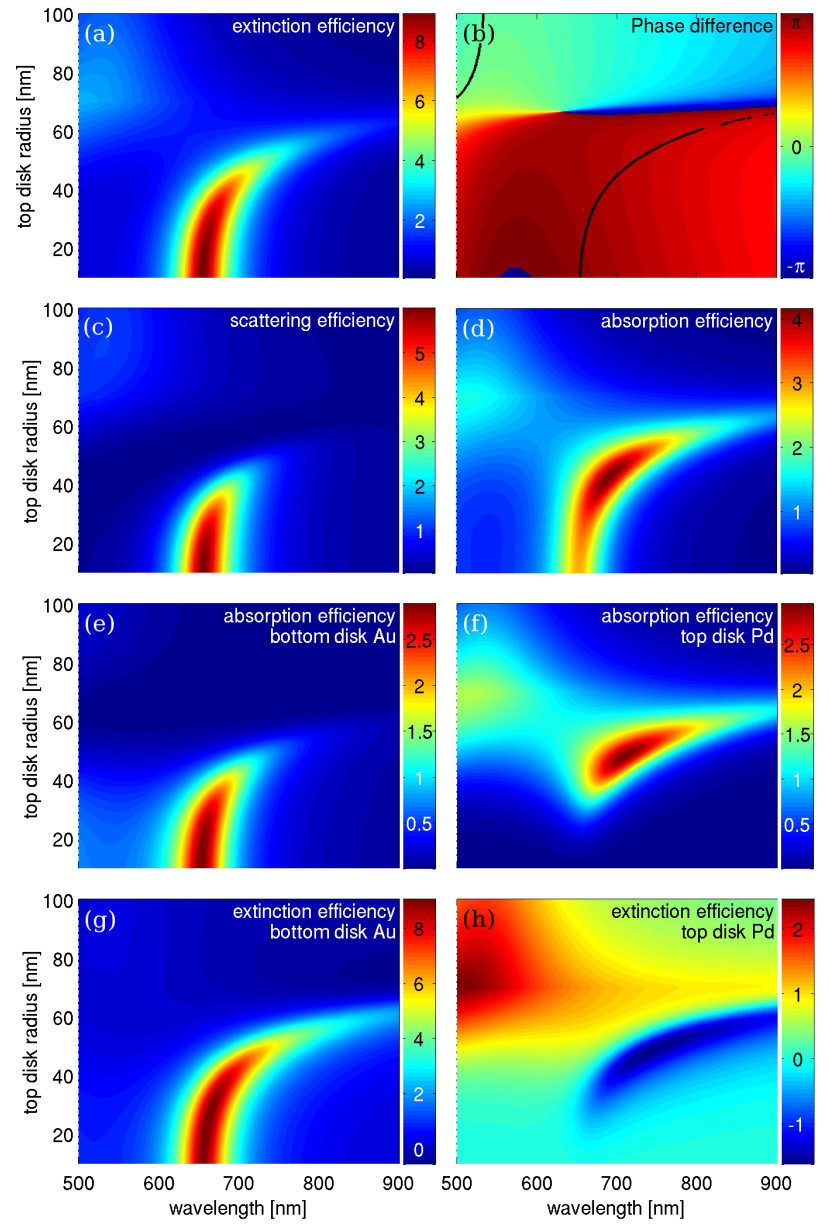

Figure 6: Optical spectra of a dipole modeled Au-Pd nanosandwich as function of wavelength and top disk radius. The bottom disk radius is set to $70 \mathrm{~nm}$ : (a) extinction efficiency; (b) phase difference between induced dipole moments; (c) scattering efficiency; absorption efficiencies of the whole nanosandwich in (d), bottom Au disk only in (e) and in top Pd disk only in (f); extinction efficiencies in bottom and top disks only in $(\mathrm{g})$ and $(\mathrm{h})$, respectively. The dipole separation is 60 nm. Notice the qualitative agreement with the Au-Pd FDTD calculated spectra in Fig. 3 such as lack of a clear distinction into a bonding and antibonding mode and the Au-driven absorption enhancement in the top Pd disk. At maximum absorption, the Pd disk efficiency is about 3, while the extinction efficiency is about 0.5 , which means that the Pd disk dissipated $c a$. six times more energy than it receives from the exciting beam. 
$r_{t}$ approaches $70 \mathrm{~nm}$ and coupling between the disks increases, which causes a transition of losses from $\mathrm{Au}$ to $\mathrm{Pd}$. Also, at short wavelengths, an absorption maximum appears, similar to what is seen in the FDTD simulations.

The origin of the two maxima is different and can be understood from an analysis of extinction in both disks. Extinction is a measure of power loss of the incident beam due to its interaction with the dipoles and is always positive, as energy can not be created in the object. The split of extinction into two parts, one coming from $\mathrm{Au}$ and the other from $\mathrm{Pd}$, demonstrates their relative contributions. We see in Fig. $6 \mathrm{~g}$ that the bottom disk is mainly responsible for removing energy from the incident beam. To a large extent, this is happening only at the main resonance, which redshifts with increasing $r_{t}$ and whose efficiency is falling from 9 to about 2 with increasing $\lambda$. However, both total scattering and absorption of the Au disk drop drastically already at $700 \mathrm{~nm}$, when extinction in Au still equals 8. Due to coupling, this excess power from $\mathrm{Au}$ is transferred to the Pd disk, where most of it is absorbed, yielding a maximum absorption efficiency of 3 at $\lambda=700 \mathrm{~nm}$. At this wavelength (and, indeed, for a narrow wavelength range too) extinction of the Pd nanodisk is negative. This means, that the Pd part of the nanosandwich returns more energy to the electromagnetic field than it receives from it. ${ }^{32}$

Conservation of energy dictates, that this extra-reradiated power at those frequencies must come from somewhere. In this case it comes from the Au nanodisk, which scatters/couples a lot of power that it has captured from the incident field. Indeed, whenever the Pd part of the nanostructure has negative extinction, the Au disk has a positive extinction and, moreover, its value is larger than the absolute value of extinction of Pd. Thus, conservation of energy is maintained, as the total nanosandwich has extinction greater than zero. Hence, our analysis clearly proves that the absorption enhancement in $\mathrm{Pd}$ is the result of radiative transfer of energy from the Au nanoantenna to the Pd disk. Moreover, this resonant transfer is connected to the bonding mode with the dipole moments being mostly out-of-phase.

The second maximum of Pd absorption occurs at short wavelength when the disk radius is approximately $70 \mathrm{~nm}$. In contrast to the first one, here transfer of energy from one dipole to the 
other is negligible and the palladium absorbs (and radiates) the energy it removes from the incident beam. Thus, at this resonance large enhancement is not observed.

In summary, in the CDA analysis we have shown the physical reason for the large enhancement in absorption in the Pd disk, namely efficient transfer of energy in the nanosandwich from the bottom Au disk element to the top Pd one.

\section{Conclusions}

We have investigated how optical absorption in a Pd nanodisk located in the "top" position of a heterometallic sandwich structure depends on the coupling to the bottom Au disk in the structure. One of these disks, the Au one, plays the role of a large nanoantenna which collects incident radiation and transfers a large amount of it to the Pd disk. We have shown, that this process is so efficient, that the Pd disk is able to absorb up to eight times more energy from the solar spectrum when in the sandwich compared to when being alone. The exact enhancement is a function and combination of the disk separation and Pd disk radius. Maximum enhancement decreases as the disks are moved away from each other and as the radius of the Pd disk becomes larger. There are two regimes of enhancement - one for small Pd disks, where the large Au nanoantenna drives directly the absorber by an enhanced near-field, and the second one, where the transfer of energy occurs when the two disks form a coupled mode. We note that the outlined conclusions are generic and directly applicable to other $\mathrm{Au}$ - (or Ag- and Al-) -lossy transition metal combinations.

\section{Relation to mechanical system}

A simple analysis of the system discussed in the main text can be carried out in terms of coupled harmonic oscillators. We start from Eqs. (1) and (2) and a simple free electron model for the permittivity $\varepsilon_{i}$ of the two dipoles $\varepsilon_{i}=1-\omega_{i}^{2} /\left(\omega\left(\omega+i \Gamma_{i}\right)\right)$, where $\omega_{i}$ is the plasma frequency and $\Gamma_{i}$ determines damping. In this approximation the quasistatic polarizability of the two dipoles

placed in vacuum is equal to $\alpha_{i}(\omega)=\varepsilon_{0} V_{i} \omega_{i}^{2} /\left(\omega_{i}^{2} L_{i}-\omega^{2}-i \omega \Gamma_{i}\right)$ (now in SI units). We rewrite 
Eqs. (1) and (2) by substituting these values and obtain

$$
\begin{aligned}
\left(-\omega^{2}-i \omega \Gamma_{1}+\omega_{1}^{2} L_{1}\right) \mathbf{P}_{1}= & \\
= & \varepsilon_{0} V_{1} \omega_{1}^{2}\left(\mathbf{E}_{0,1}-\mathbf{A}_{12} \cdot \mathbf{P}_{2}\right), \\
\left(-\omega^{2}-i \omega \Gamma_{2}+\right. & \left.\omega_{2}^{2} L_{2}\right) \mathbf{P}_{2}= \\
& =\varepsilon_{0} V_{2} \omega_{2}^{2}\left(\mathbf{E}_{0,2}-\mathbf{A}_{21} \cdot \mathbf{P}_{1}\right),
\end{aligned}
$$

where

$$
\begin{aligned}
& \mathbf{A}_{i j} \cdot \mathbf{P}_{j}=\frac{1}{4 \pi \varepsilon_{0}} e^{i k r_{i j}}\left(k^{2} \frac{\mathbf{n}_{i j} \times\left(\mathbf{n}_{i j} \times \mathbf{P}_{j}\right)}{r_{i j}}+\right. \\
& \left.\left(1-i k r_{i j}\right) \frac{\mathbf{P}_{j}-3 \mathbf{n}_{i j}\left(\mathbf{n}_{i j} \cdot \mathbf{P}_{j}\right)}{r_{i j}^{3}}\right)\left.\right|_{i \neq j},
\end{aligned}
$$

where $r_{i j}$ is the length of the vector $\mathbf{r}_{i j}$ from dipole $j$ to $i$ and $\mathbf{n}_{i j}=\mathbf{r}_{i j} / r_{i j}$.

The left-hand side of the above equations is now written in a form that allows an easy transformation into the time domain. However, the right-hand side is not and the Fourier Transform (FT) would introduce phase shifts in the terms there. Since we intend to sketch a mechanical picture of the problem at hand, we assume that $\mathbf{E}_{0,1}=\mathbf{E}_{0,2} \equiv \mathbf{E}_{0}$, which gives a symmetric field acting onto the two coupled harmonic oscillators. The position of the dipoles, with respect to the incident field and each other, means that certain terms in Eq. (5) simplify to $\mathbf{n}_{i j} \times\left(\mathbf{n}_{i j} \times \mathbf{P}_{j}\right)=-\mathbf{P}_{j}$ and $\mathbf{n}_{i j} \cdot \mathbf{P}_{j}=0$. Thus the expression for the dipole interaction reduces to $\mathbf{A}_{i j}=\frac{1}{4 \pi \varepsilon_{0}}\left(-k^{2} / r_{i j}+\left(1-i k r_{i j}\right) / r_{i j}^{3}\right) e^{i k r_{i j}}$. Keeping only the near-field term and setting $r_{i j} \equiv d$ we obtain $\left(\mathbf{A}_{12}=\mathbf{A}_{21}\right) \mathbf{A} \approx e^{i k d} / d^{3}$, where the exponential term keeps track of retardation and introduces a time shift in the FT. Assuming that retardation is negligible, we now perform the FT to obtain time dependent equations for the coupled harmonic oscillators $\left(-i \omega \mathfrak{f}(\omega) \stackrel{\mathrm{FT}}{\longrightarrow} \partial_{t} f(t)\right)$ where 
the $3 \mathrm{D}$ equations are now reduced to a $1 \mathrm{D}$ case

$$
\begin{aligned}
& \ddot{p}_{1}+\Gamma_{1} \dot{p}_{1}+\omega_{1}^{2} L_{1} p_{1}=\varepsilon_{0} V_{1} \omega_{1}^{2}\left(E_{0}-\frac{p_{2}}{4 \pi \varepsilon_{0} d^{3}}\right), \\
& \ddot{p}_{2}+\Gamma_{2} \dot{p}_{2}+\omega_{2}^{2} L_{2} p_{2}=\varepsilon_{0} V_{2} \omega_{2}^{2}\left(E_{0}-\frac{p_{1}}{4 \pi \varepsilon_{0} d^{3}}\right) .
\end{aligned}
$$

We express the dipole moment as $p_{i}=q_{i} x_{i}$, where $x_{i}$ is the displacement of a charge $q_{i}=N_{i} e$ and $N_{i}$ is the number of electrons of charge $e$ comprising dipole $i$. The plasma frequency of material $i$ is described by the free electron model $\omega_{i}^{2}=\frac{n_{i} e^{2}}{\varepsilon_{0} m_{i}}$, where $n_{i}$ is the free electron density and $m_{i}$ is the effective mass of an electron. Introducing $\omega_{i}$ into the above equations we can writen

$$
\begin{aligned}
& \ddot{x}_{1}+\Gamma_{1} \dot{x}_{1}+\left(\omega_{1}^{2} L_{1}+\frac{K}{M_{1}}\right) x_{1}+\frac{K}{M_{1}}\left(x_{2}-x_{1}\right)=\frac{1}{M_{1}} F_{1}, \\
& \ddot{x}_{2}+\Gamma_{2} \dot{x}_{2}+\left(\omega_{2}^{2} L_{2}+\frac{K}{M_{2}}\right) x_{2}+\frac{K}{M_{2}}\left(x_{1}-x_{2}\right)=\frac{1}{M_{2}} F_{2},
\end{aligned}
$$

where we have introduced $K \equiv \frac{N_{1} N_{2} e^{2}}{4 \pi \varepsilon_{0} d^{3}}, M_{i} \equiv N_{i} m_{i}$, and $F_{i} \equiv N_{i} e E_{0}$. In the preceding equations one can easily identify the expression for two mechanical oscillators, each consisting of a mass attached to a spring, and coupled to each other by a third spring $(K)$. In this formalism the equivalent spring constants of the individual disks are $k_{i} \equiv \frac{N_{i}^{2} e^{2} L_{i}}{\varepsilon_{0} V_{i}}=\frac{q_{i}^{2}}{\alpha_{i}(0)}$, where $\alpha_{i}(0)$ is the static polarizability.

In Fig. 7 we show power transfer and dissipation plots calculated from Eqs. (8) and (9). This illustrates how in a system of two coupled resonators, of which one has a narrow resonance (Au) and the other a broad one (Pd), power can be transferred from the former to the latter. In fact, the transfer is so effective that the equivalent Pd oscillator dissipates more than ten times the energy it receives just from the driving force.

\section{Acknowledgement}

We acknowledge support from the Swedish Foundation for Strategic Research via the metamaterial project SSF RMA08 (T.J.A., S.P.A., and C.W.) and the Swedish Research Council project 2010- 


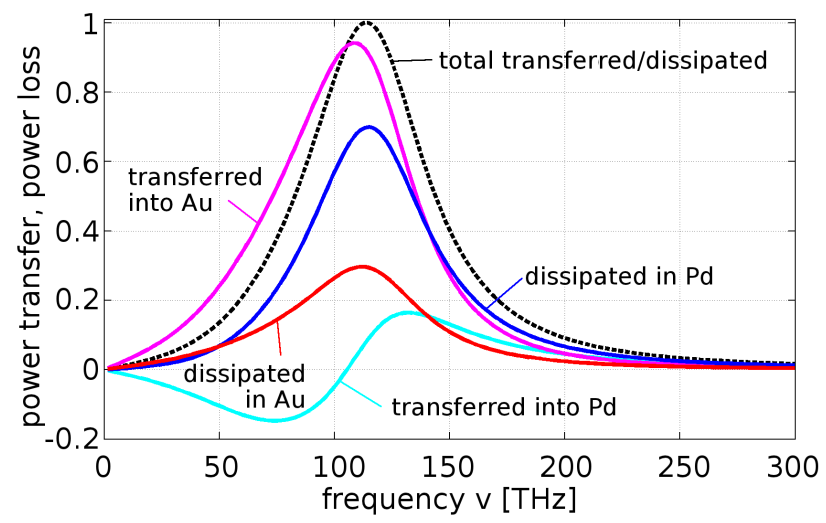

Figure 7: Relation between power transferred into and dissipated in the mechanical equivalent of a heterometallic Au-Pd sandwich. The solid curves show the power transferred into the mechanical oscillators - cyan into $\mathrm{Pd}$, magenta into $\mathrm{Au}$, and power dissipated - blue in $\mathrm{Pd}$, red in $\mathrm{Au}$. The total power transfered into the system and the dissipated power are equal (thick dashed black curve). The losses in Pd at the peak are more than 5 times the amount of power transferred directly into it from the outside force. This shows the existence of an efficient power transfer from one of the resonators to the other. The parameters used to plot this result are: $V_{\mathrm{Pd}}=6.7 \times 10^{-23} \mathrm{~m}^{3}$, $V_{\mathrm{Au}}=2.1 \times 10^{-22} \mathrm{~m}^{3}, n_{\mathrm{Pd}}=6.7 \times 10^{28} \mathrm{~m}^{-3}, n_{\mathrm{Au}}=5.9 \times 10^{28} \mathrm{~m}^{-3}, m_{\mathrm{Pd}}=5.1 \times 10^{-29} \mathrm{~kg}, m_{\mathrm{Au}}=$ $3.6 \times 10^{-29} \mathrm{~kg}, d=50 \mathrm{~nm}, L_{\mathrm{Pd}}=0.15, L_{\mathrm{Au}}=0.09, \Gamma_{\mathrm{Pd}}=10^{15} \mathrm{~s}^{-1}, \Gamma_{\mathrm{Au}}=10^{14} \mathrm{~s}^{-1}$. These parameters determine the resonance frequencies of the the diss: $\mathrm{Pd}-120 \mathrm{THz}, \mathrm{Au}-106 \mathrm{THz}$. The power transferred/dissipated are both normalized to the maximum value of the total transfered power. 
4041 Nanoplasmonics for Nanomaterials Science (C.L.).

\section{References}

(1) Englebienne, P. Analyst 1998, 123, 1599-1603.

(2) Malinsky, M. D.; Kelly, K. L.; Schatz, G. C.; Van Duyne, R. P. J. Am. Chem. Soc. 2001, 123, 1471-1482.

(3) Nath, N.; Chilkoti, A. Anal. Chem. 2002, 74, 504-509.

(4) Anker, J. N.; Hall, W. P.; Lyandres, O.; Shah, N. C.; Zhao, J.; Van Duyne, R. P. Nature Mater. 2008, 7, 442-453.

(5) Ritchie, R. H. Phys. Rev. 1957, 106, 874-881.

(6) Raether, H. In Surface Plasmons; Höhler, G., Ed.; Springer-Verlag, 1998.

(7) Apell, P.; Östling, D.; Mukhopadhyay, G. Solid State Commun. 1993, 87, 219-222.

(8) Lagarkov, A. N.; Sarychev, A. K. Phys. Rev. B 1996, 53, 6318-6336.

(9) Willingham, B.; Brandl, D.; Nordlander, P. Appl Phys B 2008, 93, 209-216.

(10) Apell, S. P.; Echenique, P. M.; Ritchie, R. H. Ultramicrosc. 1996, 65, 53-60.

(11) Cubukcu, E.; Kort, E. A.; Crozier, K. B.; Capasso, F. Appl. Phys. Lett. 2006, 89, 093120.

(12) Noginov, M. A.; Zhu, G.; Belgrave, A. M.; Bakker, R.; Shalaev, V. M.; Narimanov, E. E.; Stout, S.; Herz, E.; Suteewong, T.; Wiesner, U. Nature 2009, 460, 1110-1113.

(13) Tang, L.; Kocabas, S. E.; Latif, S.; Okyay, A. K.; Ly-Gagnon, D.-S.; Saraswat, K. C.; Miller, D. A. B. Nature Photon. 2008, 2, 226-229.

(14) Chang, C.-C.; Sharma, Y. D.; Kim, Y.-S.; Bur, J. A.; Shenoi, R. V.; Krishna, S.; Huang, D.; Lin, S.-Y. Nano Lett. 2010, 10, 1704-1709. 
(15) McFarland, A. D.; Van Duyne, R. P. Nano Lett. 2003, 3, 1057-1062.

(16) Evlyukhin, A. B.; Bozhevolnyi, S. I.; Pors, A.; Nielsen, M. G.; Radko, I. P.; Willatzen, M.; Albrektsen, O. Nano Lett. 2010, 10, 4571-4577.

(17) Shegai, T.; Langhammer, C. Adv. Mater. 2011, 23, 4409-4414.

(18) Antosiewicz, T. J.; Apell, S. P.; Claudio, V.; Käll, M. Opt. Express 2012, 20, 524-533.

(19) Christopher, P.; Xin, H.; Linic, S. Nature Chem. 2011, 3, 467-472.

(20) Linic, S.; Christopher, P.; Ingham, D. B. Nature Mater. 2011, 10, 911-921.

(21) Apell, P.; Zdunek, J.; Gillbro, T. Physica Scripta 1992, 45, 57-61.

(22) Thimsen, E.; Le Formal, F.; Grätzel, M.; Warren, S. C. Nano Lett. 2011, 11, 35-43.

(23) Persson, B. N. J. Surf. Sci. 1993, 281, 153-162.

(24) Denzler, D. N.; Frischkorn, C.; Hess, C.; Wolf, M.; Ertl, G. Phys. Rev. Lett. 2003, 91, 226102.

(25) Hendrich, C.; Bosbach, J.; Stietz, F.; Hubenthal, F.; Vartanyan, T.; Träger, F. Appl. Phys. B 2003, 76, 869-875.

(26) Brus, L. Acc. Chem. Res. 2008, 41, 1742-1749.

(27) Wadell, C.; Antosiewicz, T. J.; Langhammer, C. Nano Lett. 2012,

(28) Liu, N.; Mesch, M.; Weiss, T.; Hentschel, M.; Har, Nano Lett. 2010, 10, 2342-2348.

(29) Tittl, A.; Mai, P.; Taubert, R.; Dregley, D.; Liu, N.; Giessen, H. Nano Lett. 2011, 11, 43664369.

(30) http://rredc.nrel.gov/solar/spectra/am1.5/, NREL reference solar spectrum irradiance: air mass 1.5 .

(31) http://www.lumerical.com/tcad-products/fdtd/. 
(32) Bohren, C.; Huffman, D. Absorption and scattering of light by small particles; John Wiley and Sons, Inc., New York, 1983.

(33) Johnson, P.; Christy, R. Phys. Rev. B 1972, 6, 4370-4379.

(34) Johnson, P. B.; Christy, R. W. Phys. Rev. B 1974, 9, 5056-5070.

(35) Dmitriev, A.; Pakizeh, T.; Käll, M.; Sutherland, D. S. Small 2007, 3, 294-299.

(36) Jackson, J. D. Classical Electrodynamics, 3rd ed.; John Wiley and Sons, Inc., New York, 1999.

(37) Langhammer, C.; Kasemo, B.; Zorić, I. J. Chem. Phys. 2007, 126, 194702.

(38) Pakizeh, T.; Dmitriev, A.; Abrishamian, M. S.; Granpayeh, N.; Käll, M. J. Opt. Soc. Am. B 2008, 25, 659-667.

(39) Langhammer, C.; Yuan, Z.; Zorić, I.; Kasemo, B. Nano Lett. 2006, 6, 833-838.

(40) Draine, B. T. Astrophys. J. 1988, 333, 848-872.

(41) Draine, B. T.; Flatau, P. J. J. Opt. Soc. Am. A 1994, 11, 1491-1499.

(42) Jensen, T.; Kelly, L.; Lazarides, A.; Schatz, G. C. J. Cluster Sci. 1999, 10, 295-317.

(43) Moroz, A. J. Opt. Soc. Am. B 2009, 26, 517-527. 


\section{Graphical TOC Entry}

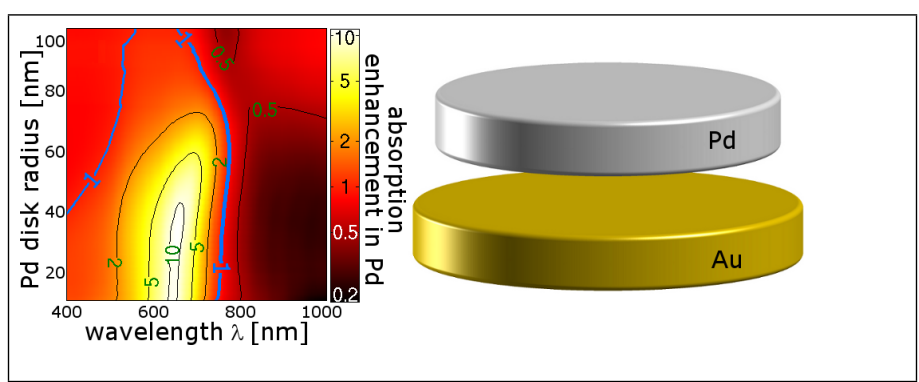

